University of Wollongong

Research Online

Australian Institute for Innovative Materials -

Papers

Australian Institute for Innovative Materials

$1-1-2013$

Quantifying fibronectin adhesion with nanoscale spatial resolution on glycosaminoglycan doped polypyrrole using Atomic Force Microscopy

Amy Gelmi

University of Wollongong, aag446@uowmail.edu.au

Michael J. Higgins

University of Wollongong, mhiggins@uow.edu.au

Gordon G. Wallace

University of Wollongong, gwallace@uow.edu.au

Follow this and additional works at: https://ro.uow.edu.au/aiimpapers

Part of the Engineering Commons, and the Physical Sciences and Mathematics Commons

Research Online is the open access institutional repository for the University of Wollongong. For further information contact the UOW Library: research-pubs@uow.edu.au 


\title{
Quantifying fibronectin adhesion with nanoscale spatial resolution on glycosaminoglycan doped polypyrrole using Atomic Force Microscopy
}

\begin{abstract}
The interaction of ECM proteins is critical in determining the performance of materials used in biomedical applications such as tissue regeneration, implantable bionics and biosensing. Methods: To improve our understanding of ECM protein-conducting polymer interactions, we have used Atomic Force Microscopy (AFM) to elucidate the interactions of fibronectin (FN) on polypyrrole (PPy) doped with different glycosaminoglycans. Results: We were able to classify four main types of FN interactions, including those related to 1) non-specific adhesion, 2) protein unfolding and subsequent unbinding from the surface, 3) desorption and 4) interactions with no adhesion. FN adhesion on PPy/hyaluronic acid showed a significantly lower density of surface adhesion with the adhesion restricted to nodule structures, as opposed to their peripheries, of the polymer morphology. In contrast, PPy/chondroitin sulfate showed a significantly higher density of surface adhesion to the point where the distribution of adhesion effectively masked the topography. Through conductive AFM imaging, we found that the conductive regions correlated with regions of FN adhesion. Conclusions: Given that the conductivity requires doping of the polymer, these findings suggest that FN adhesion is mediated by interactions with chondroitin sulfate and hyaluronic acid at the polymer surface and may be indicative of specific interactions due to contributions from electrostatic attraction between the FN and sulfate/anionic groups of the dopants. General significance: This study demonstrates the ability of AFM to resolve the protein-conducting polymer interactions at the molecular and nanoscale level, which will be important for interfacing these polymer materials with biological systems. This article is part of a Special Issue entitled Organic Bioelectronics Novel Applications in Biomedicine.
\end{abstract}

\section{Keywords}

atomic, force, microscopy, quantifying, doped, polypyrrole, fibronectin, adhesion, nanoscale, spatial, resolution, glycosaminoglycan

\section{Disciplines}

Engineering | Physical Sciences and Mathematics

\section{Publication Details}

Gelmi, A., Higgins, M. J. \& Wallace, G. G. (2013). Quantifying fibronectin adhesion with nanoscale spatial resolution on glycosaminoglycan doped polypyrrole using Atomic Force Microscopy. BBA: General Subjects, 1830 (9), 4305-4313. 
Quantifying Fibronectin Adhesion with Nanoscale Spatial Resolution on Glycosaminoglycan Doped Polypyrrole using Atomic Force Microscopy

A. Gelmi, M. J. Higgins* \& G. G. Wallace*

ARC Centre of Excellence for Electromaterials Science (ACES),

Intelligent Polymer Research Institute (IPRI),

AlIM Facility, Innovation Campus,

University of Wollongong,

Squires Way, Fairy Meadow, NSW, 2519, Australia

\section{Received Date}

Title Running Head: Fibronectin Adhesion on Glycosaminoglycan Doped Polypyrrole.

Dr Michael Higgins

Email:mhiggins@uow.edu.au

Tel: $\quad+61-2-4221-3989$

Fax: $\quad+61-2-4221-3114$

Prof. Gordon Wallace

Email:gwallace@uow.edu.au

Tel: $\quad+61-2-4221-3127$

Fax: $\quad+61-2-4221-3114$ 


\section{Abstract}

BACKGROUND: The interaction of ECM proteins is critical in determining the performance of materials used in biomedical applications such as tissue regeneration, implantable bionics and biosensing.

METHODS: To improve our understanding of ECM protein - conducting polymer interactions, we have used Atomic Force Microscopy (AFM) to elucidate the interactions of fibronectin (FN) on polypyrrole (PPy) doped with different glycosaminoglycans.

RESULTS: We were able to classify four main types of FN interactions, including those related to non-specific adhesion, protein unfolding and subsequent unbinding from the surface, desorption and interactions with no adhesion. FN adhesion on PPy/hyaluronic acid showed a significantly lower surface adhesion density with the adhesion restricted to the nodule structures, as opposed to their peripheries, of the polymer morphology. In contrast, PPy/chondroitin sulfate showed a significantly higher surface adhesion density to the point where the uniform distribution of the adhesion effectively masked the topography. Through conductive AFM imaging, we found that the conductive regions correlated with regions of FN adhesion.

CONCLUSIONS: Given that the conductivity requires doping of the polymer, these findings suggest that FN adhesion is mediated by interactions with chondroitin sulfate and hyaluronic acid exposed at the polymer surface and may be indicative of specific interactions due to contributions from electrostatic attraction between the FN and sulfate/anionic groups on the dopants.

GENERAL SIGNIFICANCE: This study demonstrates the ability of AFM to resolve the protein conducting polymer interactions at the bulk, molecular and nanoscale level, which will be important for interfacing these polymer materials with biological systems. 


\section{Introduction}

Living cells secrete extracellular matrix (ECM) proteins, such as fibronectin, vitronectin and laminin, to support adhesion, migration, proliferation and differentiation, and other processes (e.g. assembly of ECM in fibrillogenesis and mechanotransduction) that are important for cell function ${ }^{1}$. The biological function of these proteins is often played out directly at the interface, or effectively 'sandwiched', between the extracellular membrane and substratum. Their initial adsorption, adherence and subsequent conformation are critical to the cell interactions and as such the effect of different surface chemistries and materials on ECM protein interactions has significantly featured in many studies ${ }^{2,3,4}$. While the importance of ECM protein interactions is well recognized, this has been emphasized in recent times by the role they play in regulating stem cell niches ${ }^{5}$. In these niches, bound ECM protein and other growth factors, not just soluble molecules, are increasingly implicated as critical regulators of spatial and temporal determination of stem cell fate.

Understanding the interactions of proteins will be particularly important for the development of biomaterials such as conducting polymers that are a focus of this special journal issue and emerging in a wide range of biological applications ${ }^{6}$, including electrodes ${ }^{7}$ and electrode coatings for cell culture systems (e.g. for rapid cell expansion) ${ }^{8}$, organic transistors ${ }^{9}$, neural prosthesis (e.g. cochlear electrodes) ${ }^{10}$, tissue regeneration devices (e.g. nerve conduits) ${ }^{11,12}$ and stem cell scaffolds ${ }^{13}$. While the focus on ECM protein interactions in the aforementioned applications has been limited, it is already very apparent that as electrode materials conducting polymers have unique properties that are highly suited to incorporating proteins and/or controlling protein interactions ${ }^{14}$. These include the ease of chemically or biologically functionalizing the polymer backbone, the incorporation of biological dopants such as glycosaminoglycans (GAGs) (e.g. heparin, hyaluronic acid and chondroitin sulfate) that specifically bind, and imbibe, ECM proteins such as fibronectin, and electrical stimulation that can dynamically and reversibly control surface energy and protein adhesion ${ }^{14}$.

Similar to other classes of polymers and biomaterials, the surface chemistry- and redoxdependent conformation of major ECM protein such as fibronectin is thought to underlie the ability to control cell adhesion and migration via conducting polymers. The prelude to this work was demonstrated by the ability to electrically control cell adhesion using conducting polymers ${ }^{15,16}$. More recently, several groups have undertaken studies that implicate the role 
of ECM proteins in mediating cell interactions with conducting polymers ${ }^{17,18}$. Electrical stimulation of PEDOT:PSS films prior to cell seeding has shown that the oxidation state of the polymer affects cell viability ${ }^{17}$. Reduced PEDOT:PSS promoted cellular adhesion and proliferation of epithelial MDCK cells, while oxidation of PEDOT:PSS resulted in cell detachment and death. It was proposed that FN protein presents an unfavourable conformation that inhibits access to the cell-binding RGD sequence on the oxidized polymer, however the situation is opposite for the reduced polymer. A potential gradient along PEDOT:PSS film and its effect on FN adsorption and conformation has provided a welldesigned experimental approach to better understand the relationship between the proteinmaterial interaction and resulting cell behaviour such as adhesion and migration ${ }^{18,19}$. For example, 3T3-L1 fibroblast-adipose cells deposited on a PEDOT:tosylate polymer that had a potential bias of $-1 \mathrm{~V}$ to $+1 \mathrm{~V}$ distributed with a preference for cell adhesion toward the positive bias end. It was found that the amount of protein adsorption decreased ${ }^{18,19}$ but had a greater propensity to adopt a more unfolded conformation ${ }^{20}$ along the gradient toward the positive bias end of increased cell adhesion. A similar effect was observed with neural stem cells on a PEDOT:tosylate electrode with a 2-fold increase in cell adhesion on the oxidized polymer even though the protein adsorption was lower compared to the reduced polymer ${ }^{21}$. Again, the protein conformation, rather than density, was suggested to be responsible for promoting cell adhesion. Recently, the presence of $\mathrm{FN}$ in a more open confirmation on oxidized PPy/dextran sulfate films was shown using $\mathrm{QCM}^{22}$.

In many of the above studies on conducting polymers, the ensemble of the protein interaction, or bulk processes of protein adsorption, is measured using fluorescence-based approaches $^{17,18,19}$ and $\mathrm{QCM}^{22}$. The bulk information is then often extrapolated down to interactions at the molecular level, which may be difficult. By using Atomic Force Microscopy (AFM), the interaction of proteins can be directly probed the single molecule level to give insight into biophysical processes such as binding kinetics ${ }^{23}$ and force-induced conformations ${ }^{24}$. Single molecule studies take into account the multiplicity of protein interactions, especially in the case of large, modular FN protein that can exist in compact, semi-compact and extended conformations ${ }^{25}$, as well as having polyampholyte characteristics that makes it easily deformable on high charge density surfaces ${ }^{26}$. Furthermore, the complex surface properties of conducting polymers, due to different oxidation states ${ }^{27}$, inhomogenous 
doping $^{28}$ and phase separation of properties (e.g. surface potential and conductivity) ${ }^{29,30}$, that have been shown to display nanoscale lateral variation are expected to amplify variations in the conformation and adhesion of individual proteins across the polymer surface. Whilst the above studies have focused on conformation ${ }^{17,20,21}$, the FN-polymer interfacial forces are also interest as they play an important role in force-dependent signal transduction processes such as cellular forces exerted on $\mathrm{FN}$ through cell receptors and intracellular proteins to regulate cell function.

In this study, we use AFM to elucidate the different interactions, and quantify the adhesion, of FN on polypyrrole doped with glycosaminoglycans (GAGs) such as chondroitin sulfate, hyaluronic acid and dextran sulfate, which are known to have an affinity for binding FN. We specifically investigate the effect of the dopants on the prevalence of the different interactions and for spatially resolving FN adhesion as a function of nanoscale lateral variation in topography and conductivity across the polymer surface. 


\section{Materials and Methods}

\section{Reagents}

The pyrrole monomer was obtained from Merck and distilled prior to use. The chemicals used as the dopants were the sodium salts of pTS, HA, DS and CS. CS and DS were obtained from Sigma, pTS from Merck and HA from Fluka. All solutions were prepared with deionised Milli-Q water (18.2M $\Omega$ ). The functionalization chemicals 3-ethoxydimethylsilylamine propyl (3-EDSPA), gluteraldehyde (GAH) and human plasma FN were obtained from Sigma Aldrich. Phosphate buffered saline (PBS) was prepared at pH 7 in Milli-Q water (18.2M $\Omega$ ). The 3-EDSPA was prepared as a $1 \%$ solution in toluene. The GAH was prepared as a $2.5 \%$ solution in $\mathrm{pH} 7 \mathrm{PBS}$ buffer. The $\mathrm{FN}$ was prepared as a $10 \mu \mathrm{g} / \mathrm{mL}$ solution in $\mathrm{pH} 7 \mathrm{PBS}$ buffer.

\section{Preparation of polymer films}

Gold coated mylar was firstly prepared by cutting into strips of $0.5 \mathrm{~cm}$ by $2 \mathrm{~cm}$ area and then cleaned with methanol and Milli-Q water. An aqueous monomer solution of $0.2 \mathrm{M}$ pyrrole and $2 \mathrm{mg} / \mathrm{mL}$ of the counterion dopant was degassed in $\mathrm{N}_{2}$ for 10 min prior to polymerisation of the polymers. PPy films were grown galvanostatically at a current density of $0.25 \mathrm{~mA} / \mathrm{cm}^{2}$ for $10 \mathrm{~min}$ in the aqueous monomer solution using an eDAQ EA161 potentiastat. Polymer growth was performed in a standard 3-electrode electrochemical cell with the gold coated mylar as the working electrode, a platinum mesh counter electrode and $\mathrm{Ag} / \mathrm{AgCl}$ reference electrode. After growth, the films were washed with Milli-Q water, gently dried with $\mathrm{N}_{2}$ gas and placed in petri dishes until use.

\section{Protein functionalization of AFM tip}

The tip is functionalized using an aminosilzation method to covalently bind the FN to the tip. Silicon nitride (SiN) Nanoworld PNP-DB tips are used for this method due to the availability of silicon oxide groups on the surface. The tips were initially cleaned with a plasma cleaner to remove any impurities or functionalized groups on the surface. Once cleaned the tips were immediately functionalized to minimise any contaminants on the surface. The tips were placed into the EDSPA solution at room temperature for $1 \mathrm{~h}$. The tips were then removed, washed consecutively with toluene, then PBS solution. The tips were then encapsulated with the GAH solution for $1 \mathrm{~h}$, then rinsed with PBS solution. The tips were finally encapsulated in the FN solution for $1 \mathrm{~h}$, then rinsed and refrigerated in PBS solution until use.

\section{Atomic Force Microscopy - force measurements and mapping}

The force measurements were performed using an MFP-3D Asylum Research AFM (Santa Barbara, CA) and carried out in PBS fluid using a $500 \mathrm{~nm}$ approach, $0.5 \mathrm{~Hz}$ scan rate, $1 \mathrm{sec}$ dwell toward and $1 \mathrm{nN}$ trigger 
force. Single force measurements were performed with 5 consecutive measurements at one $x-y$ point, with a rest of 3 seconds, across 5 different points on the polymer surface over three individual samples for a total of 225 force curves. This process of collecting force measurements was performed on PPy films prepared with the four dopants. Force mapping was performed in PBS fluid using a $1 \mu \mathrm{m}$ approach, $0.5 \mathrm{~Hz}$ scan rate, $1 \mathrm{sec}$ dwell toward, $1 \mathrm{sec}$ dwell away and $1 \mathrm{nN}$ trigger force. The force maps were conducted over a $500 \mathrm{~nm} \times 500 \mathrm{~nm}$ and $250 \mathrm{~nm} \times 250 \mathrm{~nm}$ area on each of the polymer samples with a resolution of 32 x 32 force curves. The topographical image was calculated by returning the -max value of the data and the adhesion image was calculated by returning the difference of the average of the last 10 point at the minimum of the force curve. Analysis of the force curves were carried out using the Asylum AFM software in IGOR PRO (Wavemetrics). 


\section{Results/Discussion}

During the force measurements, the FN-functionalized AFM tip is brought into contact with the polymer to initiate binding and then the FN-polymer adhesion forces acting on the cantilever are measured as it is withdrawn. We were able to classify four main types of interactions between the FN and differently doped PPy films, as shown by the different profiles of the force curves (Fig. 1). Figure 1 shows representative examples along with a schematic describing the corresponding FN-polymer interaction. Force curves that showed no hysteresis between the approach (light grey line) and retract (dark line) curves were indicative of no adhesion between the FN and polymers (Fig. 1A). The most prevalent type of force curve showed hysteresis in the form of a significant peak at zero tip-surface separations (i.e. directly at the surface) in the retract curve, indicating an interaction involving $\mathrm{FN}$ adhesion to the polymers (Fig. 1B). The strength of protein adhesion is given as the peak maximum. This type of adhesion is typically due to the interaction of several proteins on the tip, involving both the breaking of intra and inter-protein bonds, and their subsequent detachment from the surface (Fig. 1B, schematic). Inter-protein interactions with the polymer surface may include electrostatic, hydrophobic and hydrogen bonding, while intra-protein interactions include unfolding of the protein or adhesion between the proteins, all of which may contribute to the strength or overall energy of protein adhesion. Even though the peak maximum quantifies protein adhesion to the surface, this type of interaction is generally referred in AFM measurements as being 'nonspecific' adhesion as the specific forces (e.g. hydrophobic or electrostatic) involved are not readily identified. For AFM tips functionalized with proteins, the non-specific adhesion is often always present due to the inevitable direct tip-surface interaction and as such is initially observed in the other main types of interactions in Figure 1. Again, whilst being referred to as non-specific adhesion, this interaction has been often used to determine the strength of protein adhesion to different polymer films ${ }^{31}$.

After initial, non-specific adhesion, a significant number of force curves showed hysteresis consisting of multiple, smaller peaks, or 'saw-tooth' patterns, that persist for longer tip-surface separation distances (Fig. 1C). These successive peaks, defined here as 'multiple rupture peaks', are typically due to increases in force required to sequentially unfold, or break bonds within, FNIII domains of a protein(s) that remains tethered between the tip and surface after overcoming non-specific adhesion. The tethered protein is generally "stretched" in the normal direction to the surface and applied tensile forces cause the individual domains within the protein to unfold. AFM studies on force-induced unfolding of FN III domains have shown that repeating distances of $28 \mathrm{~nm}$ between the rupture peaks corresponds to the fully unfolded length of a single FNIII domain $(\approx 70 \text { peptides } \times 0.4 \mathrm{~nm}=28 \mathrm{~nm})^{24}$. If unfolding of the FNIII domain proceeds through an intermediate pathway, smaller peak spacings of $12 \mathrm{~nm}$ are observed ${ }^{32}$. We recently 
confirmed the characteristic unfolding of FNIII domains when using AFM to measure FN protein interactions on PPy doped with glycosaminoglycans ${ }^{33}$, thus a detailed analysis of the multiple rupture peaks was not repeated in this study. Multiple rupture peaks may also occur due to the forces required to detach the protein (from the surface) at multiple binding sites along its length or involvement of multiple interacting proteins.

The last type of force curve shows that protein adhesion can occur with a constant force that is independent of the extension length (Fig. 1D). Such adhesion is referred to as 'plateau forces' and commonly observed for polyelectrolyte chain desorption from a surface, much like a polymer chain being 'peeled' off the surface. Plateau forces arise due to dependencies on the dissociation rate of repeating polymer chain-surface bonds relative to the rate at which the chain is pulled from the surface and presence of oppositely charged surfaces ${ }^{34,35}$. In contrast to the interactions involving multiple rupture peaks (Fig. 1C, schematic) where the FN molecule is generally tethered at two points and then extended in a direction normal to the surface, plateau forces indicate that the $\mathrm{FN}$ is adhered to the polymer surface along it lengths by repeating bonds (Fig. 1D, schematic). The presence of two plateaus in Figure 1D suggests an interaction with multiple proteins. Assuming that two proteins are involved, the higher force of $450 \mathrm{pN}$ is recorded as both proteins simultaneously detach from the surface but decreases by half when only one of the proteins remains adhered to the surface. A maximum tip-surface separation distance of $175 \mathrm{~nm}$ for the plateau force (total length of the protein just prior to detaching from the surface) corresponds to the fully extended length of $\mathrm{FN}^{36,37,33}$, suggesting that the repeating $\mathrm{FN}$-polymer bonds occurred along a majority of the protein's length.

In summary, the four main types of FN interactions include those of 1) non-specific adhesion, 2) protein unfolding and subsequent unbinding from the surface, 3) desorption and 4) interactions where no adhesion was observed. The prevalence of each type of interaction as a function of the dopant is displayed in Table 1. Compared to the other dopants that were similar (<3\%), PPy/HA showed the highest occurrence (17\%) of force curves with no adhesion, indicating that the probability of FN adhesion to the PPy/HA films was significantly less. All dopants showed a similar occurrence of non-specific adhesion (34-37\%), with the pTS dopant highest at 45\%. For the occurrence of multiple rupture peaks, both PPy/CS and PPy/DS were similar (63\% and $64 \%)$ and significantly higher than HA (47\%) and pTS (52\%). The occurrence of multiple rupture peaks for all dopants was significantly higher compared to their respective plateau forces $(<15 \%)$, suggesting that FN-polymer binding and subsequent stretching of the protein involves significantly fewer bonds that tether the protein to the surface, as depicted in the schematics (cf. Fig. $1 \mathrm{C}$ and D). 
Histograms of the non-specific adhesion (given as the force at peak maximum in Figure $1 \mathrm{~B}$ and $\mathrm{C}$ ) for the differently doped polymers are shown in Figure 2. Analysing the forces as histograms can reveal more information such as the most probable force distribution and number of molecules/bonds participating in the interaction. For example, the occurrence of multiple distributions with increasing peak values, or namely quantization of the adhesion force, typically correlates with an increase in the (n) number of interacting molecules. A similar observation is evident for the PPy/CS and PPy/HA films as both consist of two different peak distributions (Fig. $2 \mathrm{~A}$ and B). The most probable distribution, which occurs in the nanonewton range, is indicative of forces for the non-specific adhesion involving multiple FN proteins. In contrast, the peak values of the lower distributions are $\approx 262 \mathrm{pN}$ and $153 \mathrm{pN}$ for PPy/CS (Fig. 2A) and PPy/HA (Fig. 2B), respectively, indicating that the interaction is on the order of single molecule interactions. It has previously been possible to discern single molecule $\mathrm{FN}$ interactions with conducting polymers by analysing only the forces of the last rupture peak ${ }^{33}$. This is because the last rupture peak typically corresponds to the detachment of one, or at most two, proteins that remain tethered to the tip, as most of the other interacting proteins have already detached at shorter tip-sample separation distances (i.e. during the non-specific adhesion). Previously measured single molecule FN binding forces for PPy/CS $(164 \pm 10.1 \mathrm{pN})$ and PPy/HA $(108 \pm 8.5 \mathrm{pN})$ by analysis of the last rupture peak ${ }^{33}$ are of similar order to peak values of the lower distributions in Figure $2 \mathrm{~A}$ and $2 \mathrm{~B}$, suggesting that single protein interaction may even be detected when analysed as non-specific adhesion. This is not the case however for PPy/DS (Fig. 2C) and PPy/pTS (Fig. 2D) where no force distributions on the order of single protein interactions are observed. If one assumes that an increase in force scales with the (n) number of interacting molecules, the number of FN molecules involved in the non-specific adhesion for the higher force distribution can be roughly estimated. Therefore, $\approx 4$ proteins may be involved for both PPy/HA and PPy/CS. Gaussian fitting of the higher force distributions indicated that the maximum strength of non-specific adhesion to the differently doped polymer films increased in the order of PPy/DS $(0.53 \pm 0.12 \mathrm{nN}$; mean \pm s.d; $n=105)<$ PPy/HA $(0.63 \pm 0.21 \mathrm{nN}$; mean \pm s.d; $n=126)<$ PPy/CS $(1.03 \pm 0.09 \mathrm{nN} ;$ mean \pm s.d; $n=158)<$ PPy/pTS $(1.22$ $\pm 0.22 \mathrm{nN}$; mean \pm s.d; $\mathrm{n}=193$ ) (Fig. 2). Contact angle (CA) measurements in previous studies on these films ${ }^{38}$ where the CA increased in the order of PPy/CS $\left(13.4 \pm 2.6^{\circ}\right)<$ PP/HA $\left(18.4 \pm 2.5^{\circ}\right)<$ PPy/DS $(22.0 \pm$ $\left.0.66^{\circ}\right)<$ PPy/pTS $\left(63.2 \pm 5.5^{\circ}\right)$ indicates the protein can adhere strongest to the most hydrophilic (PPy/CS) and hydrophobic (PPy/pTS) polymers. Under physiological conditions, FN adheres more strongly in its compact conformation on hydrophobic surfaces ${ }^{39}$, which may also cause subsequent denaturation of the FN secondary structure to increase substrate binding affinity ${ }^{40}$. The compact conformation of $\mathrm{FN}$ is stabilized by intermolecular bonds ${ }^{41}$ but can be disrupted by interacting surface groups, particularly hydrophilic and negatively charged surfaces, causing the protein to adopt a more unfolded 
conformation $^{25,42}$. In this conformation, the protein can interact via different domain regions that are specific to various ECM proteins (e.g. fibronectin and collagen) and cell surface receptors. The different doped films also have varying surface roughness, ranging from $\approx 6-100 \mathrm{~nm}$ RMS (over 10 um area), that is a mitigating factor known to affect protein-biomaterial interactions ${ }^{43}$. Nanoscale surface roughness particularly influences the adsorption of large proteins such as FN and fibrinogen ${ }^{43,44}$, however, its effects are varied and generalization of relationship between roughness and protein adsorption is difficult. Recent QCM studies on FN adsorption to PPy/DS showed increased adsorption on more hydrophilic films with higher dopant concentration yet no surface morphology-induced effects on the protein adsorption was observed $^{22}$. An important point to consider is that in contrast to techniques that measure bulk processes of the protein adsorption processes, the confinement of the protein(s) at the end of the AFM tip reduces the interaction area to lengthscales well below or equivalent to the surface roughness. This is effectively analogous to measurements on the order of single proteins interaction where the influence of surface roughness is significantly reduced or negligible. In addition, AFM not only measures protein adhesion contributions from possible short- and long-range attractive forces but also the forces required to overcome additional bonds that a formed after the protein has already adhered and perhaps altered its conformation on the surface.

To further assess difference in the strength of adhesion between the more hydrophilic films doped with GAGs, we performed 'force mapping' with the FN functionalized tips to spatially map the dependence of adhesion on topography of PPy/CS and PPy/HA films. In contrast to the above force curves taken at random single $\mathrm{X}-\mathrm{Y}$ positions, force mapping performs an array of force curves to enable corresponding height and adhesion data to be quantified simultaneously as function of the $X-Y$ position across the surface. For height maps of PPy/CS (Fig. 3A) and PPy/HA (Fig. 3B), the lighter areas correspond to the higher regions, or nodules of the polymer, while the darker regions correspond to the lower regions of the nodule peripheries. The surface roughness of PPy/CS and PPy/HA were comparable with values of $6.5 \pm$ $6.5 \mathrm{~nm}$ (mean \pm s.d; $\mathrm{n}=1024$ ) and $6.1 \pm 6.1 \mathrm{~nm}$ (mean $\pm \mathrm{s} . \mathrm{d} ; \mathrm{n}=1024)$, respectively, which are similar to values obtained using standard AFM imaging ${ }^{45}$. While these images have relatively low resolution, the nodular topography of the polymer is still discernible. Due to the lengthy acquisition time of these images in PBS, we occasionally encountered the effect of lateral sample drift that causes skewness of the image. This is particularly evident in Fig. 3B that shows that nodule topography of PPy/HA as having a stretched appearance diagonally across the surface, however this does not affect the correlation with the adhesion map. The adhesion maps of PPy/CS (Fig. 3C) and PPy/HA (Fig. 3D) show a difference in both the strength and lateral distribution of the protein adhesion. Similarly to the histograms (Fig. 3), PPy/CS showed a higher strength of adhesion compared to PPy/HA (cf. Fig. 3C and D), as indicated by the difference in their 
scale bars. To correlate the adhesion with topography, we firstly applied a threshold to the adhesion maps to exclude pixels with values $<0.3 \mathrm{nN}$ and then overlaid them onto the corresponding height images (Fig. $3 E$ and $F$ ). The threshold was used to assess values associated with the higher force distributions in the histogram, or effectively those values of non-specific adhesion that reflect the maximum strength of protein adhesion. Due to a significantly higher occurrences of adhesion (red pixels) above this threshold across the surface for PP/CS (77\% surface coverage), there was no correlation between adhesion and topography to the point where the high density and uniform distribution of adhesion effectively masked the topography (Fig. 3E). However, some of the few remaining areas without adhesion did appear to correlate with low lying areas (darker regions) or peripheries. Conversely for PPy/HA, the adhesion was more distributed along the nodular regions (lighter areas), as opposed to peripheries, and thus occurred at much lower density (24\% surface coverage) (Fig. 3F). When the threshold of the adhesion was further increased to $0.8 \mathrm{nN}$, the correlation between the adhesion and topography for the different polymer remained the same (data not shown). The correlation between adhesion and topography was also assessed at a higher resolution across a $100 \mathrm{~nm}$ scan. Once again, there was no clear correlation for PPy/CS whereas PP/HA appeared to show higher adhesion at the nodules though some binding was evident in the peripheries (Supplementary figure 1). This was particularly evident for a single, large nodular structure in these smaller scans areas.

To similarly assess the lateral variation in adhesion but with interactions more closely associated with single molecules, we overlaid the topography with pixels (green) that represented force curves consisting of multiple rupture peaks as classified in Fig. 1c (Fig. 4). As mentioned above, these types of force curves have characteristics of single molecule interactions with the final peak representing the force required to detach a few, or often, single FN molecules from the surface. Similar to the non-specific adhesion, the overlay image for PPy/CS (Fig. 4A) showed a significantly higher density (33\% of curves or 1332 interactions per $\mu \mathrm{m}^{2}$ ) of multiple rupture peak interactions compared to PPy/HA (21\% of curves or 860 interactions per $\mu \mathrm{m}^{2}$ ) (Fig. 4B). However, no correlation between the multiple rupture peaks and topography was observed, particularly for PPy/HA which was unexpected given the observed correlation for non-specific adhesion in Figure 3F. In part this may be attributed to the increased spatial interaction area of single molecule $\mathrm{FN}$ interactions, as binding can occur at varying distances ranging from $60-170 \mathrm{~nm}$ along its contour length ${ }^{33}$. The protein may bind to the polymer at a distance beyond the pixel resolution ( 1 pixel $=15 \times 15 \mathrm{~nm}$ ) of the force maps, with the actual $x-y$ position of binding different from the recorded $x-y$ value (green pixel). This is different to the situation for non-specific adhesion where the interaction occurs at zero tip-sample separation distances and effectively localized near the position of the tip. The surface roughness of PP/CS and PPy/HA was again similar, giving calculated surface areas of $0.28 \mu \mathrm{m}^{2}$ and $0.27 \mu \mathrm{m}^{2}$, respectively, 
within each map. The difference in the density of the multiple rupture peak interactions between the polymers also correlated with the \% occurrence of these FN interactions observed in the single force curve analysis (Table 1). Histograms of the force from the final peak of each force curve (i.e. each green pixel) revealed probable distributions with widths of 50-200 pN, confirming the likelihood that single molecule FN interactions are involved in these interactions. More specifically, gaussian fitting of the distributions gave values of $122 \pm 23.5 \mathrm{pN}$ (mean \pm s.d; $\mathrm{n}=1024$ ) and $70.3 \pm 13.5 \mathrm{pN}$ (mean \pm s.d; $\mathrm{n}=1024$ ) for PPy/CS (Fig. 4C) and PPy/HA (Fig. 4D), respectively, which within the error of the standard deviation are in agreement with values obtained in our previous AFM study on FN interactions with CS and HA doped $\mathrm{PPy}^{33}$.

Conductive AFM scans were taken on PPy/CS and PPy/HA to further assess the correlation between the FN adhesion, topography and conductivity of the films (Fig. 5). PPy/HA clearly showed that regions of conductivity were confined to the nodular regions of the polymer, while the peripheries showed little or no measurable current (cf Fig. 5A and C). This correlation of the topography and conductivity is also evident in cross-sectional profiles where the current values (dashed curve) reach maximum at the nodules and are negligible at the peripheries in the topography profile (solid curve) (Fig. 5E). The conductivity of $\mathrm{PPy} / \mathrm{CS}$ is however more uniformly distributed across the surface with no clear correlation with the topography (cf. Fig. 5B and D), indicating more homogenous doping of the polymer. The extent of the homogenous doping was reflected in the calculated surface area of conductive regions (threshold > 5 pA) that was $97.7 \%$ for PPy/CS compared to 54\% for PPy/HA. Similarly, conductive AFM imaging of polybithiophene films has revealed higher conductivity in the nodules compared to the nodular periphery ${ }^{29}$. These observations are further supported by Kelvin Probe Force Microscopy (KPFM) of $\mathrm{PPy} / p \mathrm{TS}$ films showing a more negative potential at the nodules and explained by these regions as having a higher work function, or increased doping, than their peripheries ${ }^{27}$. This characteristic phase separation in surface properties (e.g. conductivity, surface potential) associated with the morphology of conducting polymers has also been revealed using AFM phase imaging. For example, both the modulus ${ }^{29}$ and surface charge/energy ${ }^{30}$ was shown to undergo phase separation.

The dependence of the non-specific adhesion on the topography is further depicted in Figure 6 showing the protein interaction with both PPy/HA (Fig. 6A) and PPy/CS (Fig. 6B) using actual 3-D height images overlaid with the corresponding conductivity where areas of black indicate low or no conductivity, while areas in green indicate higher conductivity. The schematic of the tip with functionalized protein is drawn close to scale. Whilst the morphology and roughness of the films are comparable, the density of the non-specific adhesion acquired in the force maps closely correlates with the distribution of 
conductive, or more CS or HA doped, regions across the film, suggesting that FN adhesion to the polymer is mediated by interactions with the dopants. The HA doped regions are restricted to the area of the nodules which reduces the density of non-specific adhesion, whereas the CS is more uniformly doped throughout the polymer and therefore promotes a higher density of binding. Up until now we have referred to these interactions as non-specific adhesion, however we suggest that they may actually be more indicative of specific interactions due to contributions from electrostatic attraction between the FN and sulfate/anionic groups on the dopants.

A primary biological function of GAGs is to recognize and bind ECM proteins for regulating cellular activities, including cell adhesion, extracellular matrix modelling and fibrillogenesis ${ }^{46}$. Therefore, GAG's such as HA, CS, DS and heparin sulfate (HS) are often incorporated through doping into conducting polymers with the intention of promoting favourable interactions with proteins to improve biocompatibility $^{38,45}$. For example, the interaction of heparin with the protein, fibroblast growth factor-2 (FGF-2), has been implicated in the ability to control stem cell differentiation on PEDOT/heparin films ${ }^{47}$. The inhibition of smooth muscle cell proliferation, in contrast to endothelial cells, on PPy/Hep films has been explained by the interaction of the heparin with its putative cell receptors that causes antiproliferative effects via proposed mechanisms in which cell cycle progression into $G_{1}$ is arrested through decreased activation of extracellular signal-regulated kinase ${ }^{48}$. We have recently shown that single molecule FN binding to PPy doped with GAGs occurs at several positions on the protein that correlate with the well-characterized heparin binding domains, suggesting a specific, sub-molecular interaction between FN and GAGs present at the polymer surface ${ }^{33}$. The findings in the present study further supports the involvement of the GAG dopants in FN adhesion and, furthermore, shows that type of dopant and differences in the spatial distribution of the dopant effects the magnitude of protein adhesion, density of adhesion per unit area, and locality of adhesion.

Acknowledgements: This work has been supported by the Australian Research Council under the Australian Research Fellowship and DP110104359 (Dr Michael Higgins) and ARC Federation Fellowship of Prof. Gordon Wallace. We also greatly acknowledge the Australian National Fabrication Facility (ANFF) for providing Atomic Force Microscopy instrumentation. 

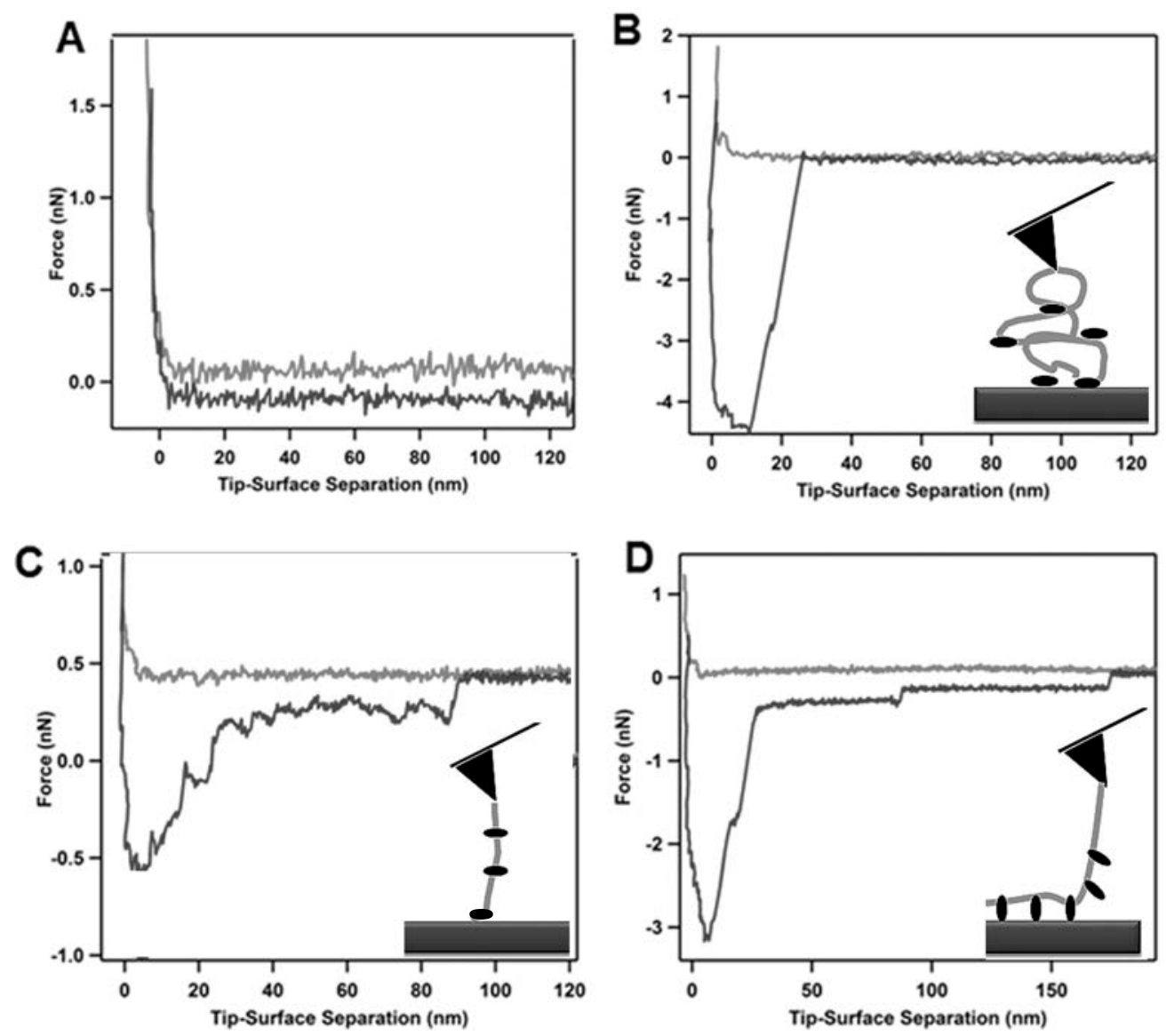

Figure 1. Force profiles showing different types of FN interactions. The light and dark curves are the tip approach and retract from the polymer surface, respectively. Insets are schematic of corresponding FN interaction. The FN protein is represented the grey line and the black ovals represent the bonds involved in the interaction. The bonds may reside within the protein and/or occur at the protein-polymer interface. The different types of FN interactions are classified as (A) No adhesion, (B) Non-Specific Adhesion, (C) Multiple Rupture Peaks and (D) Plateaus. 


\section{Dopant}

\begin{tabular}{l|llll}
\hline Type of Interaction & $\begin{array}{l}\text { Chondroitin } \\
\text { Sulfate }\end{array}$ & $\begin{array}{l}\text { Hyaluronic } \\
\text { Acid }\end{array}$ & Dextran Sulfate & $\begin{array}{l}\text { Sulfonate } \\
\text { No Adhesion }\end{array}$ \\
\hline \hline & 2 & 17 & 0 & 3 \\
Non-Specific & 34 & 36 & 37 & 45 \\
Multiple Rupture Peaks & 64 & 47 & 63 & 52 \\
Plateaus & 5 & 9 & 15 & 15 \\
\hline \hline
\end{tabular}

Table 1. Table showing the percentage (\%) occurrence of force profiles displaying each type of FN interaction on the differently doped polymers. The total number of force curves obtained for each dopant was 255 . Note: the \% of plateaus are treated separately, as they all contained either non-specific and multiple rupture peaks. 

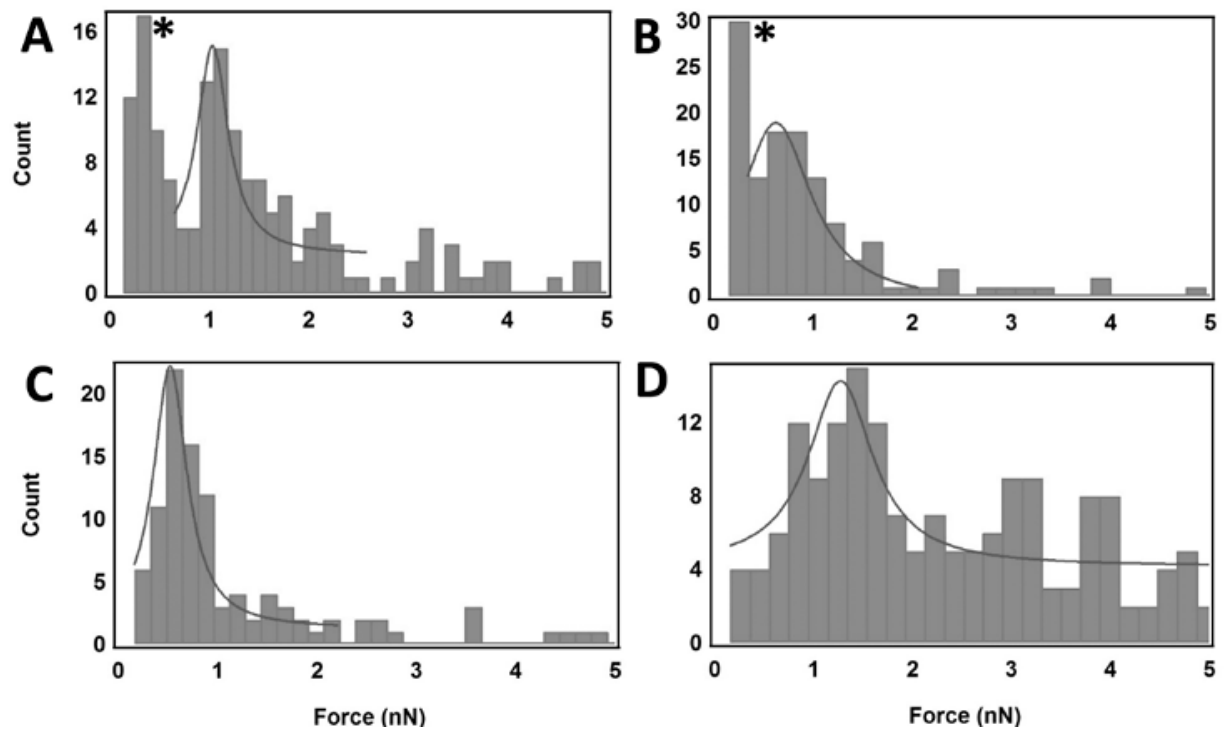

Figure 2. Histograms of maximum force of non-specific adhesion for (A) PPy/CS, (B) PPy/HA, (C) PPy/DS and (D) PPy/pTS. Solid lines are gaussian fits to the higher force distribution. Asterix denote lower force distributions for PPy/CS and PP/HA. 

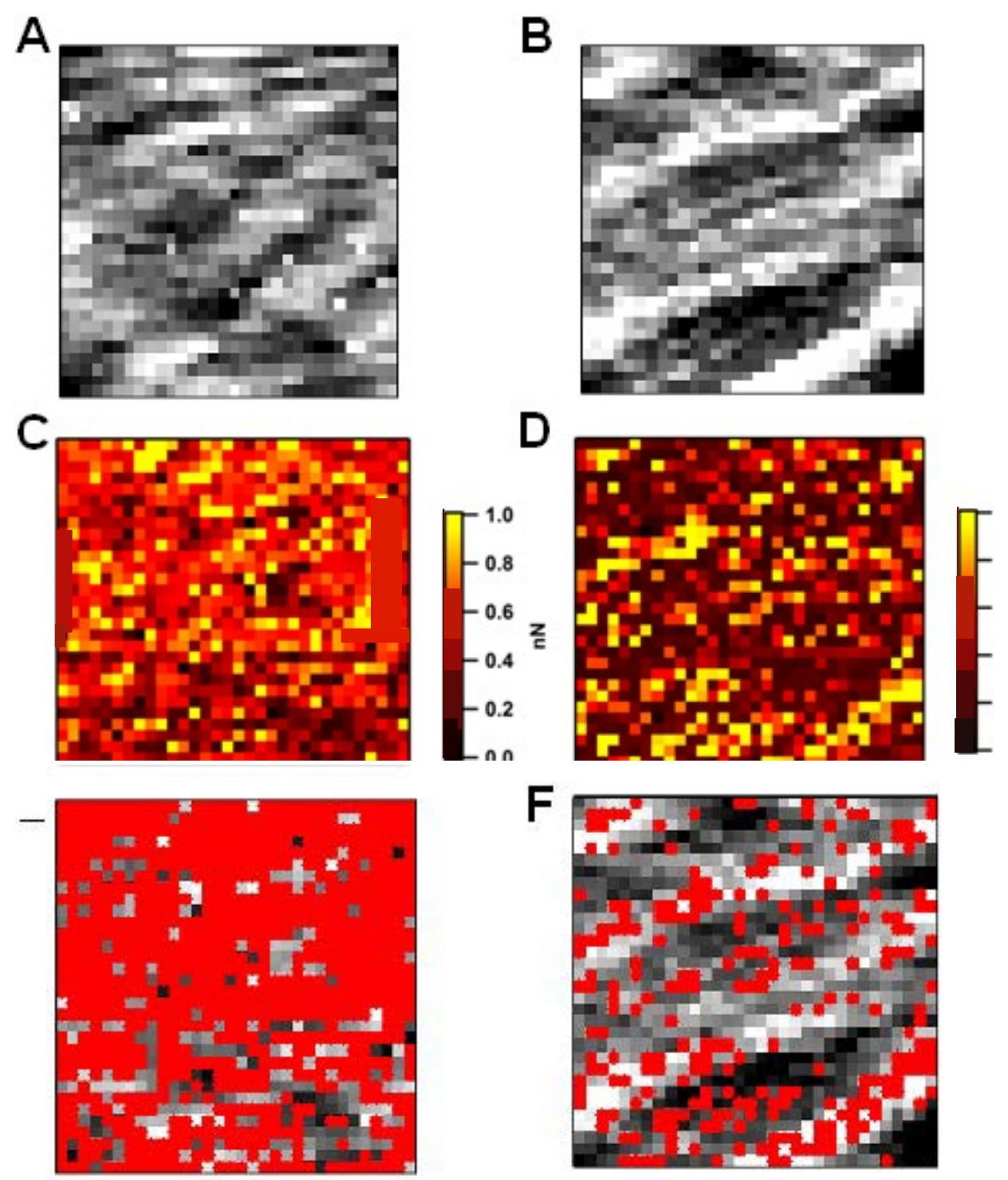

Figure 3. $500 \mathrm{~nm}$ force maps for topography (A) PPy/CS (Z scale $30 \mathrm{~nm})$ and (B) PPy/HA (Z scale $20 \mathrm{~nm})$ and adhesion (C) PPy/CS and (D)PPy/HA. A mask with a threshold of $>0.3 \mathrm{nN}$ was applied to each adhesion map and used as the overlay (red pixels) on the corresponding topography for (E) PPy/CS and (F) PPy/HA. 
A

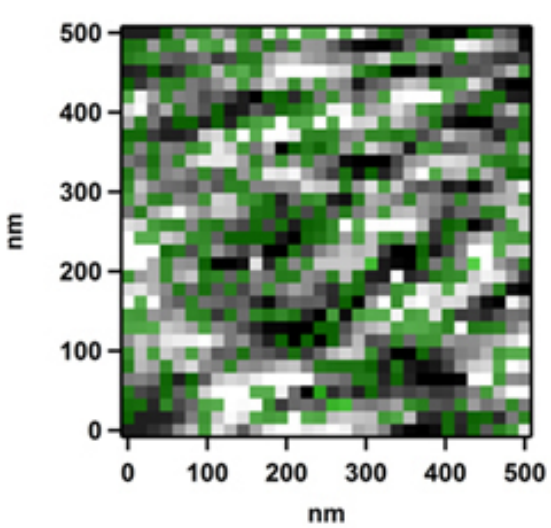

C

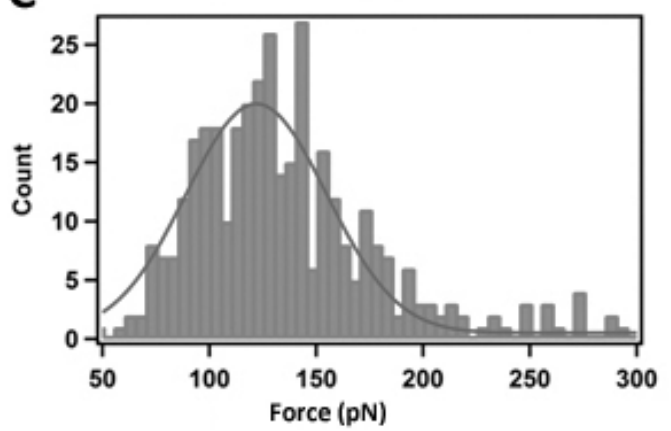

B

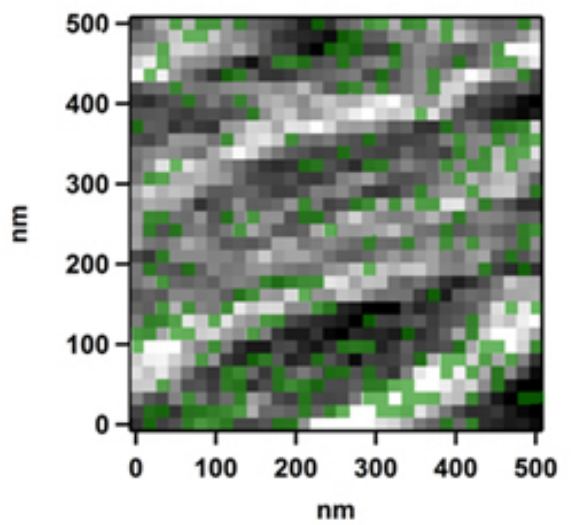

D

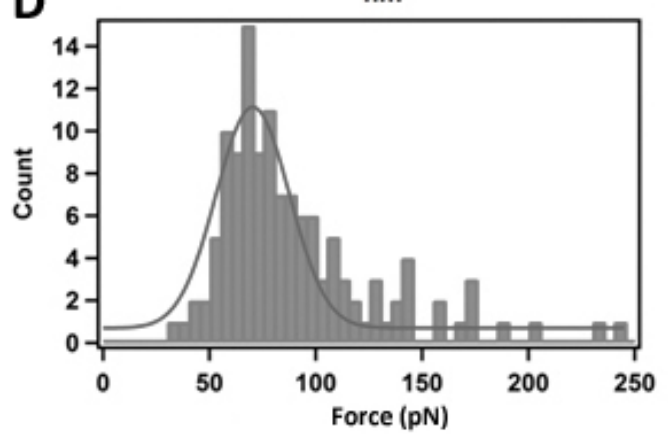

Figure 4. Multiple rupture peaks obtained from adhesion maps (green pixels) and overlaid to show their respective positions on the corresponding topography for(A) PPy/CS and (B) PPy/HA. Histograms showing the force of the final rupture peaks for (C) PPy/CS and (D) PPy/HA. Solid lines are gaussian fits. 

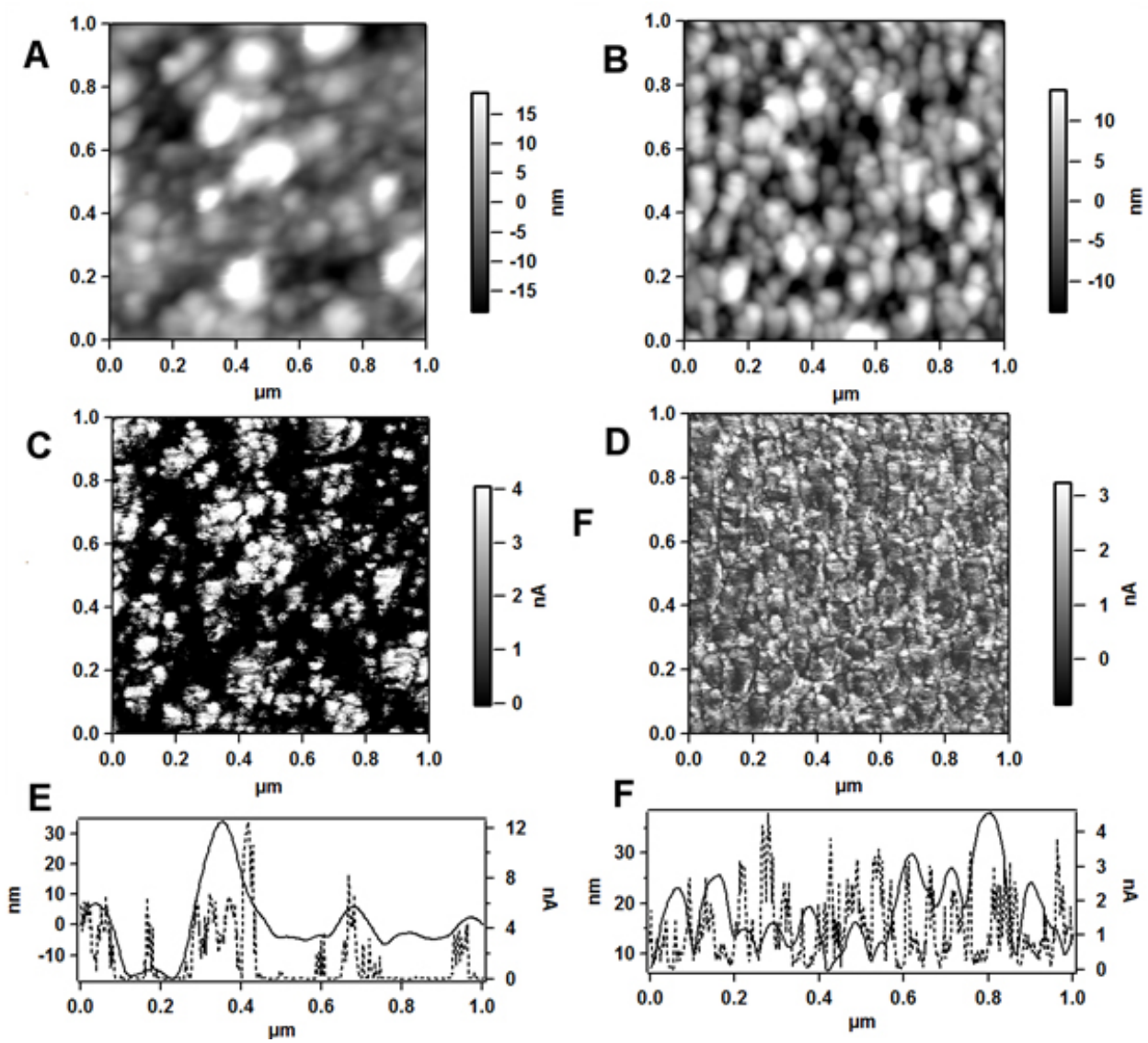

Figure 5. PPy/HA (A) topography image, (C) current image and (E) cross-section showing topography (solid) and current (dashed). PPy/CS (B) topography image, (D) current image and (F) cross-section showing topography (solid) and current (dashed). 

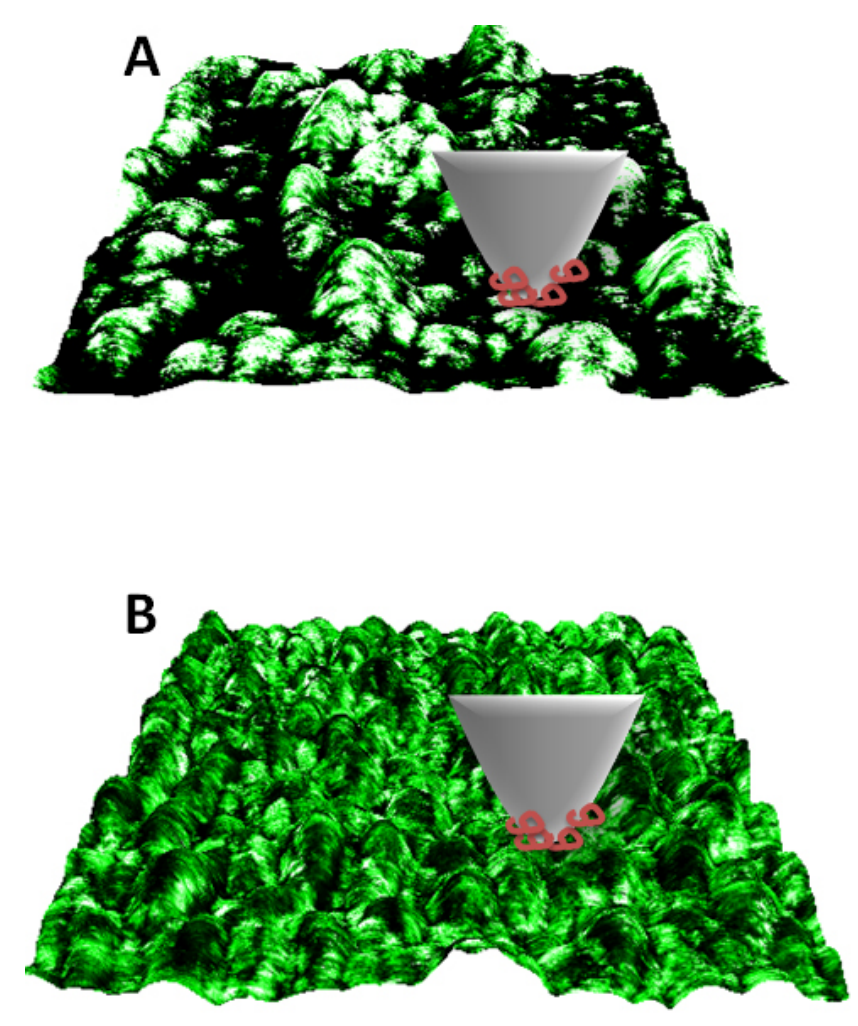

Figure 6. Schematic of protein-surface interaction using actual 3-dimensional topography with overlay of corresponding conductivity (green is conductive, black is non-conductive, Z scale $4 \mathrm{nA}$ ). Scan area is $1 \mu \mathrm{m}$ and AFM tip is drawn roughly to scale $(\approx 30 \mathrm{~nm}$ tip radius). FN is represented in red (A) HA displays inhomogenous conductivity resulting in lower probability of adhesion. The protein may not adhere to non-conductive areas (black) of the polymer. (B) CS is more homogenous giving a higher probability of FN adhesion. 


\section{References}

${ }^{1}$ Giancotti, F. G.; Ruoslahti, E., Integrin Signaling. Science 1999, 285, 1028-1033.

2 Dewez, J.-L.; Doren, A.; Schneider, Y.-J.; Rouxhet, P. G., Competitive adsorption of proteins: Key of the relationship between substratum surface properties and adhesion of epithelial cells. Biomaterials 1999, 20, 547-559.

${ }^{3}$ Chastain, S. R.; Kundu, A. K.; Dhar, S.; Calvert, J. W.; Putnam, A. J., Adhesion of mesenchymal stem cells to polymer scaffolds occurs via distinct ECM ligands and controls their osteogenic differentiation. Journal of Biomedical Materials Research Part A 2006, 78A, 73-85.

${ }^{4}$ Koenig, A. L.; Gambillara, V.; Grainger, D. W., Correlating fibronectin adsorption with endothelial cell adhesion and signaling on polymer substrates. Journal of Biomedical Materials Research Part A 2003, 64A, 20-37.

${ }^{5}$ Lutolf, M. P.; Gilbert, P. M.; Blau, H. M., Designing materials to direct stem-cell fate. 2009.

${ }^{6}$ Owens, R. M.; Malliaras, G. G., Organic Electronics at the Interface with Biology. MRS Bulletin 2010, 35, 449-456.

${ }^{7}$ Cui, X.; Wiler, J.; Dzaman, M.; Altschuler, R. A.; Martin, D. C., In vivo studies of polypyrrole/peptide coated neural probes. Biomaterials 2003, 24, 777-787.

${ }^{8}$ Persson, K. M.; Karlsson, R.; Svennersten, K.; Löffler, S.; Jager, E. W. H.; Richter-Dahlfors, A.; Konradsson, P.; Berggren, M., Electronic Control of Cell Detachment Using a Self-Doped Conducting Polymer. Advanced Materials 2011, 23, 4403-4408.

${ }^{9}$ Khodagholy, D.; Curto, V. F.; Fraser, K. J.; Gurfinkel, M.; Byrne, R.; Diamond, D.; Malliaras, G. G.; Benito-Lopez, F.; Owens, R. M., Organic electrochemical transistor incorporating an ionogel as a solid state electrolyte for lactate sensing. Journal of Materials Chemistry 2012, 22, 4440-4443.

${ }^{10}$ Richardson, R. T.; Wise, A. K.; Thompson, B. C.; Flynn, B. O.; Atkinson, P. J.; Fretwell, N. J.; Fallon, J. B.; Wallace, G. G.; Shepherd, R. K.; Clark, G. M.; O'Leary, S. J., Polypyrrole-coated electrodes for the delivery of charge and neurotrophins to cochlear neurons. Biomaterials 2009, 30, 2614-2624.

${ }^{11}$ V. R. Shastri, C. E. Schmidt, H. T. Kim, J. P. Vacanti, R. Langer. Polypyrrole--A Potential Candidate for Stimulated Nerve Regeneration Mater. Res. Soc. Proc. 1996, 414, 113-118.

12 Quigley, A. F.; Razal, J. M.; Thompson, B. C.; Moulton, S. E.; Kita, M.; Kennedy, E. L.; Clark, G. M.; Wallace, G. G.; Kapsa, R. M. I., A conducting-polymer platform with biodegradable fibers for stimulation and guidance of axonal growth. Advanced Materials 2009, 21, 4393-+.

${ }^{13}$ Pelto, J.; Björninen, M.; Pälli, A.; Talvitie, E.; Hyttinen, J. A. K.; Mannerström, B.; Suuronen Seppanen, R.; Kellomäki, M.; Miettinen, S.; Haimi, S., Novel Polypyrrole Coated Polylactide Scaffolds Enhance Adipose Stem Cell Proliferation and Early Osteogenic Differentiation. Tissue Engineering 2012.

${ }^{14}$ Higgins, M. J.; Molino, P. J.; Yue, Z.; Wallace, G. G., Organic Conducting Polymer-Protein Interactions. Chemistry of Materials 2012, 24, 828-839.

${ }^{15}$ Wong, J. Y.; Langert, R.; Ingberi, D. E., Electrically conducting polymers can noninvasively control the shape and growth of mammalian cells. Science 1994, 91, 3201-3204. 
${ }^{16}$ Schmidt, C. E.; Shastri, V. R.; Vacanti, J. P.; Langer, R., Stimulation of neurite outgrowth using an electrically. Proceeding of the National Academy of Sciences 1997, 94, 8948-8953.

${ }^{17}$ Svennersten, K.; Bolin, M. H.; Jager, E. W. H.; Berggren, M.; Richter-Dahlfors, A., Electrochemical modulation of epithelia formation using conducting polymers. Biomaterials 2009, 30, 6257-6264.

${ }^{18}$ Wan, A. M. D.; Brooks, D. J.; Gumus, A.; Fischbach, C.; Malliaras, G. G., Electrical control of cell density gradients on a conducting polymer surface. Chemical Communications 2009, 5278-5280.

${ }^{19}$ Gumus, A.; Califano, J. P.; Wan, A. M. D.; Huynh, J.; Reinhart-King, C. A.; Malliaras, G. G., Control of cell migration using a conducting polymer device. Soft Matter 2010, 6, 5138-5142.

${ }^{20}$ Wan, A. M. D.; Schur, R. M.; Ober, C. K.; Fischbach, C.; Gourdon, D.; Malliaras, G. G., Electrical Control of Protein Conformation. Advanced Materials 2012, 24, 2501-2505.

${ }^{21}$ Salto, C.; Saindon, E.; Bolin, M.; Kanciurzewska, A.; Fahlman, M.; Jager, E. W. H.; Tengvall, P.; Arenas, E.; Berggren, M., Control of neural stem cell adhesion and density by an electronic polymer surface switch. Langmuir 2008, 24, 14133-14138.

22 Molino, P. J.; Higgins, M. J.; Innis, P. C.; Kapsa, R. M. I.; Wallace, G. G., Fibronectin and Bovine Serum Albumin Adsorption and Conformational Dynamics on Inherently Conducting Polymers: A QCM-D Study. Langmuir 2012, 28, 8433-8445.

${ }^{23}$ Meadows, P. Y.; Bemis, J. E.; Walker, G. C., Single-molecule force spectroscopy of isolated and aggregated fibronectin proteins on negatively charged surfaces in aqueous liquids. Spectroscopy 2003, 9566-9572.

${ }^{24}$ Rief, M.; Gautel, M.; Gaub, H. E., Unfolding forces of titin and fibronectin domains directly measured by AFM. Advances in experimental medicine and biology 2000, 481, 129-142.

${ }^{25}$ Bergkvist, M.; Carlsson, J.; Oscarsson, S., Surface-dependent conformations of human plasma fibronectin adsorbed to silica, mica, and hydrophobic surfaces, studied with use of Atomic Force Microscopy. Journal of biomedical materials research. Part A 2003, 64, 349-56.

${ }^{26}$ Pernodet, N.; Rafailovich, M.; Sokolov, J.; Xu, D.; Yang, N. L.; McLeod, K., Fibronectin fibrillogenesis on sulfonated polystyrene surfaces. Journal of Biomedical Materials Research Part A 2003, 64, 684-692.

${ }^{27}$ Barisci, J. N.; Stella, R.; Spinks, G. M.; Wallace, G. G., Characterisation of the topography and surface potential of electrodeposited conducting polymer films using atomic force and electric force microscopies. Electrochimica Acta 2000, 46, 519- 531.

${ }^{28}$ Semenikhin, O. A.; Jiang, L.; lyoda, T.; Hasimoto, K.; Fujishima, A.; Serra Moreno, J.; Panero, S.; Materazzi, S.; Martinelli, A.; Sabbieti, M. G.; Agas, D.; Materazzi, G., Atomic Force Microscopy and Kelvin Probe Force Microscopy Evidence of Local Structural Polypyrrole-polysaccharide thin films characteristics: electrosynthesis and biological properties. Journal of Physical Chemistry 1996, 100, 9-11.

${ }^{29}$ O'Neil, K. D.; Shaw, B.; Semenikhin, O. A., On the origin of mesoscopic inhomogeneity of conducting polymers. Journal of Physical Chemistry B 2007, 9253-9269. 
${ }^{30}$ Gelmi, A.; Higgins, M. J.; Wallace, G. G., Attractive and Repulsive Interactions Originating from Lateral Nanometer Variations in Surface Charge/Energy of Hyaluronic Acid and Chondroitin Sulfate Doped Polypyrrole Observed using Atomic Force Microscopy. The Journal of Physical Chemistry B 2012, 116, 13498-505.

${ }^{31}$ Wang, M. S.; Palmer, L. B.; Schwartz, J. D.; Razatos, A., Evaluating protein attraction and adhesion to biomaterials with the atomic force microscope. Langmuir 2004, 20, 7753-7759.

32 Oberhauser, A. F.; Badilla-fernandez, C.; Carrion-vazquez, M.; Fernandez, J. M., The mechanical hierarchies of fibronectin observed with single-molecule AFM. Journal of Molecular Biology 2002, 319, 433-447.

33 Gelmi, A.; Higgins, M. J.; Wallace, G. G., Resolving Sub-Molecular Binding and Electrical Switching Mechanisms of Single Proteins at Electroactive Conducting Polymers. Small 2012 (in press).

${ }^{34}$ Haupt, B. J.; Ennis, J.; Sevick, E. M., The detachment of a polymer chain from a weakly adsorbing surface using an AFM tip. Langmuir 1999, 15, 3886-3892.

${ }^{35}$ Chatellier, X.; Senden, T. J.; Joanny, J. F.; Di Meglio, J. M., Detachment of a single polyelectrolyte chain adsorbed on a charged surface. EPL (Europhysics Letters) 2007, 41, 303.

${ }^{36}$ Erickson, H. P.; Carrell, N.; McDonagh, J. A. N., Fibronectin molecule visualized in electron microscopy: a long, thin, flexible strand. The Journal of cell biology 1981, 91, 673-678.

37 Mao, Y.; Schwarzbauer, J. E., Fibronectin fibrillogenesis, a cell-mediated matrix assembly process. Matrix Biology 2005, 24, 389-399.

${ }^{38}$ Gilmore, K. J.; Kita, M.; Han, Y.; Gelmi, A.; Higgins, M. J.; Moulton, S. E.; Clark, G. M.; Kapsa, R.; Wallace, G. G., Biomaterials skeletal muscle cell proliferation and differentiation on polypyrrole substrates doped with extracellular matrix components. Biomaterials 2009, 30, 5292-5304.

${ }^{39}$ Conti, M.; Donati, G.; Cianciolo, G.; Stefoni, S.; Samorì, B., Force spectroscopy study of the adhesion of plasma proteins to the surface of a dialysis membrane: role of the nanoscale surface hydrophobicity and topography. Journal of biomedical materials research 2002, 61, 370-379.

${ }^{40}$ Culp, L. A.; Sukenik, C. N., Cell type-specific modulation of fibronectin adhesion functions on chemicallyderivatized self-assembled monolayers. Journal of Biomaterials Science, Polymer Edition 1998, 9, 1161-1176.

41 Johnson, K. J.; Sage, H.; Briscoe, G.; Erickson, H. P., The Compact Conformation of Fibronectin Is Determined by Intramolecular lonic Interactions. Journal of Biological Chemistry 1999, 274, 15473-15479.

${ }^{42}$ Baugh, L.; Vogel, V., Structural changes of fibronectin adsorbed to model surfaces probed by fluorescence resonance energy transfer. Journal of Biomedical Materials Research Part A 2004, 69, 525-534.

43 Hovgaard, M. B.; Rechendorff, K.; Chevallier, J.; Foss, M.; Besenbacher, F., Fibronectin adsorption on tantalum: the influence of nanoroughness. The Journal of Physical Chemistry B 2008, 112, 8241-8249.

${ }^{44}$ Sela, M. N.; Badihi, L.; Rosen, G.; Steinberg, D.; Kohavi, D., Adsorption of human plasma proteins to modified titanium surfaces. Clinical oral implants research 2007, 18, 630-638. 
${ }^{45}$ Gelmi, A.; Higgins, M. J.; Wallace, G. G., Physical surface and electromechanical properties of doped polypyrrole biomaterials. Biomaterials 2010, 31, 1974-1983.

46 J. Turnbull, A. Powell, S., Guimond. Heparan sulfate: decoding a dynamic multifunctional cell regulator. Trend. Cell Biol., 2001, 11, 75-82.

${ }^{47}$ Herland, A.; Persson, K. M.; Lundin, V.; Fahlman, M.; Berggren, M.; Jager, E. W. H.; Teixeira, A. I., Electrochemical control of growth factor presentation to steer neural stem cell differentiation. Angewandte Chemie-International Edition 2011, 50, 12529-12533.

${ }^{48}$ Stewart, E. M.; Liu, X.; Clark, G. M.; Kapsa, R. M. I.; Wallace, G. G., Inhibition of smooth muscle cell adhesion and proliferation on heparin-doped polypyrrole. Acta biomaterialia 2012, 8, 194-200. 